\title{
MicroRNA-449 suppresses proliferation of hepatoma cell lines through blockade lipid metabolic pathway related to SIRT1
}

\author{
HONGYI ZHANG $^{1 *}$, ZHIQIANG FENG ${ }^{1 *}$, RUI HUANG ${ }^{2,3}$, ZHENGLIN XIA $^{2,3}$, \\ GUOAN XIANG $^{3}$ and JINQIAN ZHANG ${ }^{2}$
}

\author{
${ }^{1}$ Department of Hepatobiliary Surgery, Air Force General Hospital, Beijing 100142; ${ }^{2}$ Institute of Infectious Diseases, \\ Beijing Ditan Hospital, Capital Medical University, Beijing 100015; ${ }^{3}$ Department of General Surgery, \\ The Second People's Hospital of Guangdong Province, Southern Medical University, \\ Guangzhou 510515, P.R. China
}

Received May 27, 2014; Accepted July 15, 2014

DOI: $10.3892 /$ ijo.2014.2596

\begin{abstract}
MicroRNA (miRNA or miR) inhibition of oncogenic related pathways has been shown to be a promising therapeutic approach for cancer. SIRT1 might be a promoter factor on tumorigenesis of hepatocellular carcinoma (HCC). However, the mechanism is unknown. We investigated whether miRNAs regulate the SIRT1 and its downstream SREBP-lipogenesischolesterogenesis metabolic pathway in hepatoma cells. Human hepatoma cells were transfected with miR-449 mimics and inhibitors, and the effects of miR-449 on cell proliferation was assessed. We identified the miRNAs, miR-449, that control lipogenesis and cholesterogenesis in hepatoma cells by inhibiting SIRT1 and SREBP-1c expression and downregulating their targeted genes, including fatty acid synthase (FASN) and 3-hydroxy-3-methylglutaryl CoA reductase (HMGCR). MiR-449 repressed DNA synthesis, mitotic entry and proliferation of hepatoma cells. Restoration of miR-449 led to suppression of SIRT1 expression and liver tumorigenesis. The newly identified miRNAs, miR-449 represents a novel targeting mechanism for HCC therapy.
\end{abstract}

\section{Introduction}

Hepatocellular carcinoma (HCC) is the fifth most common cancer worldwide and among the leading causes of cancer-related

Correspondence to: Dr Jinqian Zhang, Institute of Infectious Diseases, Beijing Ditan Hospital, Capital Medical University, Beijing 100015, P.R. China

E-mail: jingwanghou@163.com

Dr Hongyi Zhang, Department of Hepatobiliary Surgery, Air Force General Hospital, Beijing 100142, P.R. China

E-mail: jingwanghou@sina.com

*Contributed equally

Key words: microRNA, SIRT1, proliferation, SREBP-1c, lipid metabolism, hepatocellular carcinoma deaths. In the majority of cases, HCC develops as a result of chronic inflammation and cirrhosis caused by viral hepatitis $\mathrm{B}$ and $\mathrm{C}$, ethanol or aflatoxins (1). HCC is associated with a very poor prognosis (2), because only a limited number of patients are eligible for potentially curative treatment options such as surgical resection followed by orthotopic liver transplantation. Therefore, there is an urgent need to develop effective therapeutic strategies for the large number of HCC patients with advanced disease.

For a long time, hepatocarcinogenesis has been considered to be the result of different genetic alterations that ultimately lead to malignant transformation (3). Nowadays, cancer development is no longer thought to be induced only by genetic and genomic alterations, but also closely associated with lipid metabolism (4). The studies of Karagozian et al indicate an adipogenic lifestyle alone is sufficient for the development of nonalcoholic steatohepatitis, hepatic stem cell activation and hepatocarcinogenesis in wild-type mice (5). In recent years, metabolism is talked highly important in cancer research, especially the lipid metabolism. Researchers believe that the de novo fatty acid synthesis plays an important role in tumor development (6). Sphingolipids, a family of membrane lipids, are bioactive molecules that participate in diverse functions controlling fundamental cellular processes such as cell division, differentiation and cell death (7). Given that most of these cellular processes form the basis for several pathologies, it is not surprising that sphingolipids are key players in several pathological processes, such as diabetes, Alzheimer's disease and hepatocellular carcinoma (7).

miRNA regulation of gene expression plays a role in development, differentiation, proliferation and apoptosis (8-13). Expressions of miRNAs are abnormal in a variety of cancers and miRNA may play a role in tumorigenesis (14-21). Several miRNA have been identified that may act as tumor suppressor genes (22-27). The microRNAs 449 are potent inducers of cell death, cell cycle arrest and/or cell differentiation. They belong to the same family as the p53-responsive microRNAs miR-34. Instead of p53, however, the cell cycle regulatory transcription factor E2F1 
induces miR-449. All members of this microRNA family are capable of mediating cell cycle arrest and apoptosis and might thereby contribute to tumor suppression. Underlying mechanisms include the downregulation of histone acetyl transferases and consecutive activation of p53, but also the targeting of cyclin dependent kinases and their association partners. Thus, miR-34 and miR-449 provide an asymmetric feedback loop to balance E2F and p53 activities. More recently, it was discovered that miR-449 helps to ensure proper cell function but also to avoid cancer, marking a close link between cell differentiation and tumor suppression $(28,29)$.

We predicted SIRT1 as miR-449 target gene. Recent data suggest that SIRT1 may function as an oncogene and play a role in tumorigenesis (30-33). Because sterol regulatory element binding protein (SREBP)-1c is a transcription factor that controls the expression of genes related to fatty acid and triglyceride synthesis in tissues with high lipid synthesis rates such as adipose tissue and liver. Previous studies indicate that SIRT1 can regulate the expression and function of SREBP-1c in liver. Recent in vivo studies have suggested a crucial role for SIRT1 in regulating SREBP-1c expression and activity in liver. In vivo, SIRT1 activation decreased expression of SREBP-1c and SREBP-1c target genes (34). Conversely, SIRT1 knockdown in liver induced the expression of SREBP-1c and its target genes encoding lipid-synthesizing enzymes (35). Furthermore, SREBP-1c deacetylation by SIRT1 on Lys-289 and Lys-309 inhibits SREBP-1c activity by decreasing its stability and its association with lipogenic target genes (34). Targeting the aberrant SREBP-lipogenesis-cholesterogenesis pathway related to SIRT1 may lead to new approaches to the treatment of HCC. MiR-449 might regulate cellular proliferation through a SIRT1-SREBP-1c pathway implicating energy metabolism; it will be revelatory for further study on the possible applications in clinical treatment for HCC.

\section{Materials and methods}

Culture cells. Human hepatoma cell lines HepG2 and Huh7 from ATCC (Manassas, VA, USA) were cultured in Dulbecco's modified Eagle's medium (DMEM) supplemented with $10 \%$ fetal bovine serum (FBS) (Gibco-BRL Co. Ltd., USA) and $1 \%$ penicillin-streptomycin-neomycin (PSN) Antibiotic Mixture (Invitrogen, Carlsbad, CA, USA) at $37^{\circ} \mathrm{C}$ in a humidified $5 \% \mathrm{CO}_{2} / 95 \%$ air environment.

Reagents. Human miRNA precursors, miRNA inhibitors, antisense oligonucleotides or small interfering RNA (siRNA), TaqMan miRNA assay, mirVana ${ }^{\mathrm{TM}}$ miRNA isolation kit and Lipofectamine 2000 were purchased from Life Technologies (Invitrogen). CellTiter 96H AQueous One Solution Cell Proliferation Assay (mitochondrial MTS assay).

Bioinformatics analysis of human SIRT1. The 3' untranslated region (3'-UTR) sequences were retrieved from the Entrez Nucleotide database (http://www.ncbi.nlm.nih.gov/nuccore). The potential miRNA targets within the conserved regions in $3^{\prime}$-UTR of SIRT1 were predicted by miRBase (www. mirbase.org/) (36).
Treatments. The expression plasmid was pcDNA 4-SIRT1 (gifts from Leonard Guarente, USA). For transfection, cells in 12-well plates were transfected with the indicated plasmids $(1 \mu \mathrm{g} / w e l l)$, miRNA mimics, antisense oligonucleotides or small interfering RNA (siRNA) at the indicated final concentrations in the culture medium using Lipofectamine 2000. After transfection for $72 \mathrm{~h}$, cells were subsequently harvested for immunoblot analysis.

Quantitative real-time reverse transcription-polymerase chain reaction ( $q R T-P C R)$. Total RNA was prepared from cells using the RNeasy Mini kit (Qiagen, Valencia, CA) and subjected to reverse transcription by SuperScriptH III reverse transcriptase (Life Technologies) according to the manufacturer's instructions. A hot start at $95^{\circ} \mathrm{C}$ for $5 \mathrm{~min}$ was followed by 40 cycles at denaturation at $95^{\circ} \mathrm{C}$ for $15 \mathrm{sec}$, annealing of the primers at $58^{\circ} \mathrm{C}$ for $30 \mathrm{sec}$ and elongation at $72^{\circ} \mathrm{C}$ for $30 \mathrm{sec}$ using ABI 7500 Fast Real-Time PCR System (Invitrogen). Data were normalized to $18 \mathrm{~S}$ rRNA or GAPDH and represented as the average fold of three independent duplicates. To determine intrinsic miR-449 expression, miRNA was prepared from cells using the mirVana ${ }^{\mathrm{TM}}$ miRNA isolation kit (Invitrogen). Mature miRNA was quantified by the TaqMan miRNA assay (Invitrogen) in accordance with the manufacturer's instructions. The data were normalized by RNU6B.

Western blot analysis. Cell lysates were prepared from miR-449, NC (miR-negative control)-transfected or non-transfected cells using a lysis buffer [50 mM Tris (pH 8), $150 \mathrm{mM}$ $\mathrm{NaCl}, 0.02 \% \mathrm{NaN}_{3}, 0.1 \%$ SDS, $1 \% \mathrm{NP}-40$ and $0.5 \%$ sodium deoxycholate] containing $1 \mathrm{mM}$ phenylmethylsulfonyl fluoride and protease inhibitor cocktail (Roche Applied Science, Indianapolis, IN). Protein concentration was determined by Bradford assay using Coomassie Plus Protein Reagent (Thermo Scientific, Rockford, IL, USA). Western blot was performed using the Novex system (Invitrogen).

Primary antibodies anti-SIRT1, SREBP-1c, FASN, HMGCR and $\beta$-actin (Santa Cruz Biotechnology, Santa Cruz, $\mathrm{CA})$, and secondary antibodies which were conjugated with horseradish peroxidase (GE Healthcare, Piscataway, NJ) were used. Detection of protein bands was done using Enhanced Chemiluminescence Western Blotting Detection Reagents.

BrdU labeling and mitotic index. BrdU labeling was used to evaluate DNA synthesis. After released from the second thymidine arrest at indicated time points, cells grown in 12-well plate were pulse labeled with $\operatorname{BrdU}(50 \mu \mathrm{M})$ for $30 \mathrm{~min}$. After three washes of PBS, cells were fixed with $1 \mathrm{ml}$ of Carnoy's fixative (3 parts methano 1:1 part glacial acetic acid) at $-20^{\circ} \mathrm{C}$ for $20 \mathrm{~min}$, and followed by three washes of PBS. Subsequently, DNA was denatured by incubation of $2 \mathrm{M}$ $\mathrm{HCL}$ at $37^{\circ} \mathrm{C}$ for $60 \mathrm{~min}$, followed by three washes in borate buffer (0.1 M borate buffer, $\mathrm{pH} 8.5)$. After incubation with the blocking buffer, cells were stained with anti-BrdU antibody (BD Biosciences, 1:100) overnight at $4^{\circ} \mathrm{C}$. After washes in PBS, cells were incubated with Texas Red-conjugated anti-mouse goat IgG for $30 \mathrm{~min}$ at RT. After washes, cells were mounted and BrdU positive cells were manually scored under an immunofluorescence microscope. 
Mitotic events were scored by time-lapse videomicroscopy and DNA staining. Cells were synchronized as described above. Real-time images were captured every 10 min with Openlab software. Mitotic events of control, cells were scored by their morphological change (from flat to round-up). For each experiment, at least 800 cells were videotaped, tracked and analyzed. Alternatively, nocodazole $(100 \mathrm{ng} / \mathrm{ml})$ was added into the medium after release, cells were collected, fixed and stained with DNA dye (Hoechst 33258). Mitotic cells were scored by nuclear morphology and DNA condensation.

Cell growth and proliferation assay. Cell growth was determined by the colorimetric tetrazolium derived XTT (sodium 3'-(1-(phenylaminocarbonyl)-3, 4-tetrazolium)-bis (4-methoxy-6-nitro) benzene sulfonic acid hydrate) assay (Roche Applied Science, Mannheim, Germany), and DNA synthesis of cells was assessed by the BrdU (bromodeoxyuridine) incorporation assay (Roche Applied Science). For the cell growth and proliferation assay, at $48 \mathrm{~h}$ after transfection of treatment, the cells of each group were re-seeded in 96-well plates at a density of $0.3-1 \times 10^{4}$ cells per well. After $48 \mathrm{~h}$, XTT and incorporated BrdU were measured colorimetrically using a microtiter plate reader at a wavelength of $450 \mathrm{~nm}$ (37).

Fatty acid and cholesterol quantification. The amounts of long chain fatty acids and cholesterols were determined using the Free Fatty Acid Quantification Kit and Cholesterol/Cholesteryl Ester Detection Kit (Abcam, Cambridge, MA) in cells transfected with miR-449 or NC. The amounts of fatty acids and cholesterols were normalized by total cell numbers. The relative fatty acid or cholesterol (\%) was assigned as $100 \%$ in NC cells.

Statistical analysis. Continuous normally distributed variables were represented graphically as mean \pm standard deviation (SD). For statistical comparison of quantitative data between groups, analysis of variance (ANOVA) or t-test was performed. To determine differences between groups not normally distributed, medians were compared using Kruskal-Wallis ANOVA. $\chi^{2}$ test was used when necessary for qualitative data. The degree of association between variables was assessed using Spearman's non-parametric correlation. All statistical analyses were carried out using SPSS software version 13.0 (SPSS Inc., Chicago, IL, USA). Probabilities of 0.05 or less were considered to be statistically significant.

\section{Results}

miR-449 inhibits mRNA expression of SIRT1, SREBPI-c and their downstream genes in hepatoma cells. To investigate whether miRNAs regulate the SIRT1 and its downstream SREBP-lipogenesis-cholesterogenesis metabolic pathway in hepatoma cells, we first used DIANA microT v4.0 online software (http://diana.cslab.ece.ntua.gr/) to predict if one or more miRNAs target SIRT1, the key factor that regulate fatty acid, lipid and cholesterol biosynthesis and homeostasis. The miR-449 was retrieved that potentially co-targeted
3'-UTRs of SIRT1 mRNAs. To further verify if miR-449 directly binds with 3'-UTRs of SIRT1, we performed 3'-UTR luciferase reporter assay and found that the relative 3'-UTR luciferase activities of SIRT1 were significantly decreased in miR-449 transfected hepatoma cells HepG2 and Huh7 (Fig. 1A and B). The results confirm that SIRT1 mRNAs are direct targets of miR-449. To validate whether the miRNAs control the SREBP-lipogenesis-cholesterogenesis pathway in hepatoma cells, we performed qRT-PCR quantification analyses. The miR-449 inhibited the mRNA expression of SIRT1 in HepG2 and Huh7 hepatoma cell lines, respectively. Expression of SREBP, FASN and HMGCR, which are downstream regulated genes of SIRT1, was decreased in mRNA levels of HepG2 and Huh7 cells (Fig. 1C and D) by miR-449, and could be upregulated by inhibitors of miR-449 (Fig. 2). The protein level of SIRT1, SREBP-1c, FASN and HMGCR was inhibited also by miR-449 in HepG2 (Fig. 3A) and Huh7 (Fig. 3B), similarly to treatment with SIRT1 siRNA (Fig. 3C and D), but were upregulated by inhibitors of miR-449 or overexpression of SIRT1 (Fig. 3).

Because SREBP1c, FASN and HMGCR are important enzymes for de novo synthesis of fatty acid and cholesterol individually (34), we subsequently examined the levels of fatty acid and cholesterol in cells. As shown in Fig. 4, the amounts of intracellular fatty acid and cholesterol were significantly decreased in miR-449-transfected HepG2 (Fig. 4A) and Huh7 (Fig. 4B) cells compared with the control groups. To further test the specificity of miR-449 for SREBP-lipogenesis-cholesterogenesis, antisense oligonucleotides against miR-449 were used as miR-449 inhibitors. By blocking endogenous miR-449 in hepatoma cells, the amounts of intracellular fatty acid and cholesterol were upregulated by inhibitors of miR-449 similarly to cells treated with overexpression of SIRT1 (Fig. 4A and B). These results suggested that miR-449 inhibitors increased SIRT1, SREBP-1c and their downstream gene expression, and miR-449 could inhibit SIRT1-SREBP signaling through reduction expression of SIRT1 and decreased the levels of fatty acid and cholesterol in hepatoma cells.

miR-449 suppresses DNA synthesis and mitotic entry in hepatoma cells. Cells were synchronized at the G1/S boundary by double thymidine block, and then released into mitosis. After $24 \mathrm{~h}, \mathrm{BrdU}$ was added into the medium at indicated time points to evaluate DNA synthesis. As shown in Fig. 5A and $\mathrm{B}$, incorporation of BrdU into the control, accumulation of mitotic HepG2 and Huh7 cells was significantly delayed in miR-449-treated cells in each point. In contrast, accumulation of mitotic HepG2 or Huh7 cells was significantly promoted at every time point by inhibitors of miR-449.

To further examine the specific effect of the overexpression of miR-449 on mitotic entry, we repeated this experiment and evaluated the mitotic entry. Overexpression miR-449 significantly delayed mitotic entry of HepG2 or Huh7 cells (Fig. 6A and B). In contrast, mitotic entry of HepG2 or Huh7 cells was significantly promoted in miR-449-treated cells at each point (Fig. 6A and B).

miR-449 restrains cell proliferation in hepatoma cells. To assess the potential for biological consequences elicited by 

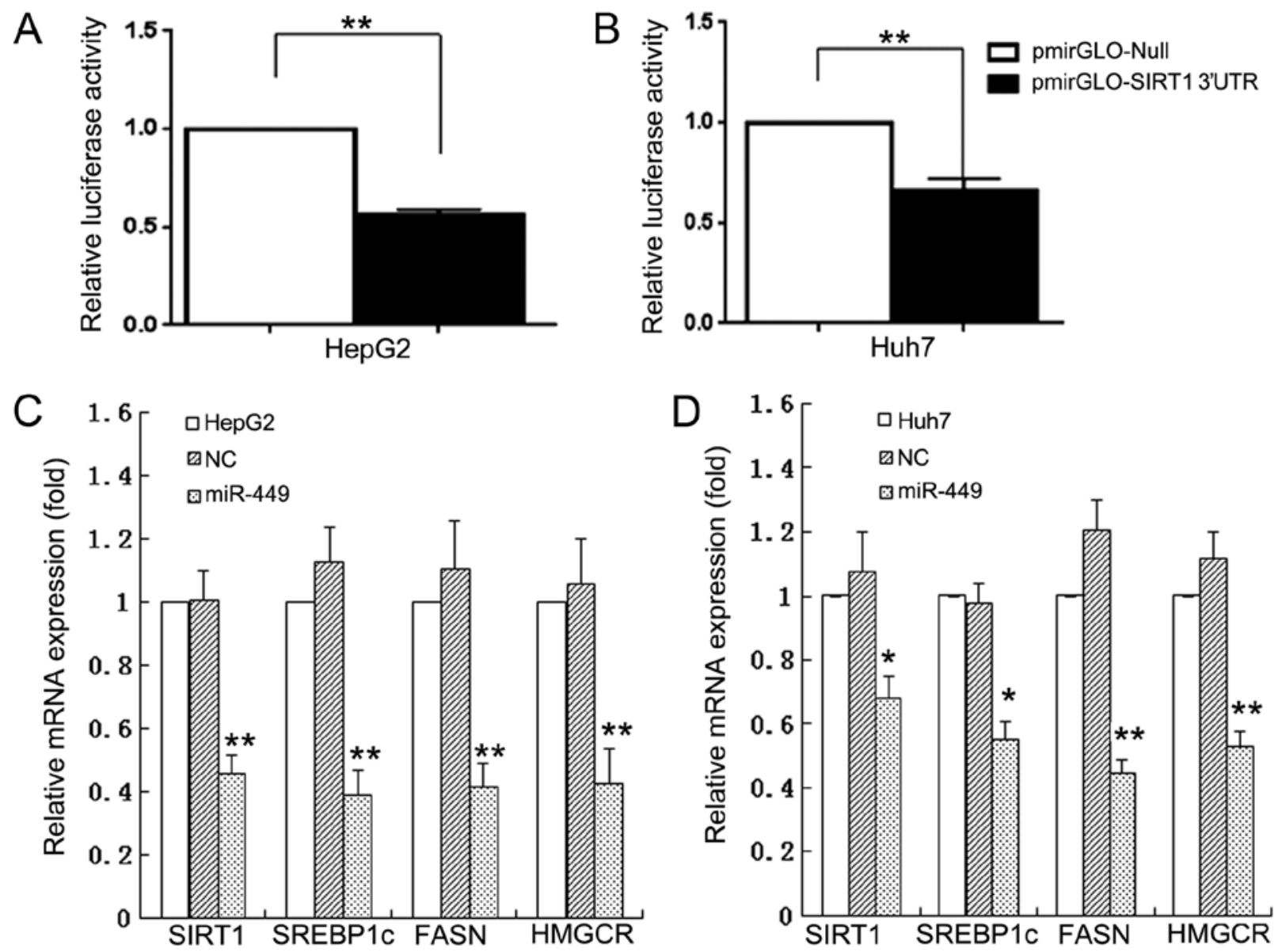

Figure 1. MiR-449 directly binds with 3'-UTRs of SIRT1, and inhibits mRNA expression of SIRT1, SREBP1-c and their downstream genes in hepatoma cell lines. To investigate whether miRNAs regulate the SIRT1 and its downstream SREBP-lipogenesis-cholesterogenesis metabolic pathway in hepatoma cells, we first used DIANA microT v4.0 online software (http://diana.cslab.ece.ntua.gr/) to predict if one or more miRNAs target SIRT1, the key factor that regulate fatty acid, lipid and cholesterol biosynthesis and homeostasis. The miRNAs, miR-449, were retrieved that potentially co-targeted 3'-UTRs of SIRT1 mRNAs. To further verify if miR-449 directly binds with 3'-UTRs of SIRT1, we performed 3'-UTR luciferase reporter assay and found that the relative 3'-UTR luciferase activities of SIRT1 were significantly decreased in miR-449 transfected hepatoma cells HepG2 and Huh7 (A and B). The results confirm that SIRT1 mRNAs are direct targets of miR-449. To validate whether the miRNAs control the SREBP-lipogenesis-cholesterogenesis pathway in hepatoma cells, we performed qRT-PCR quantification analyses. The miR-449 inhibited the mRNA expression of SIRT1 in HepG2 and Huh7 hepatoma cell lines, respectively. The mRNA expression of SREBP, FASN and HMGCR, which are downstream regulated genes of SIRT1, was decreased in mRNA levels of HepG2 and Huh7 cells (C and D) by miR-449.
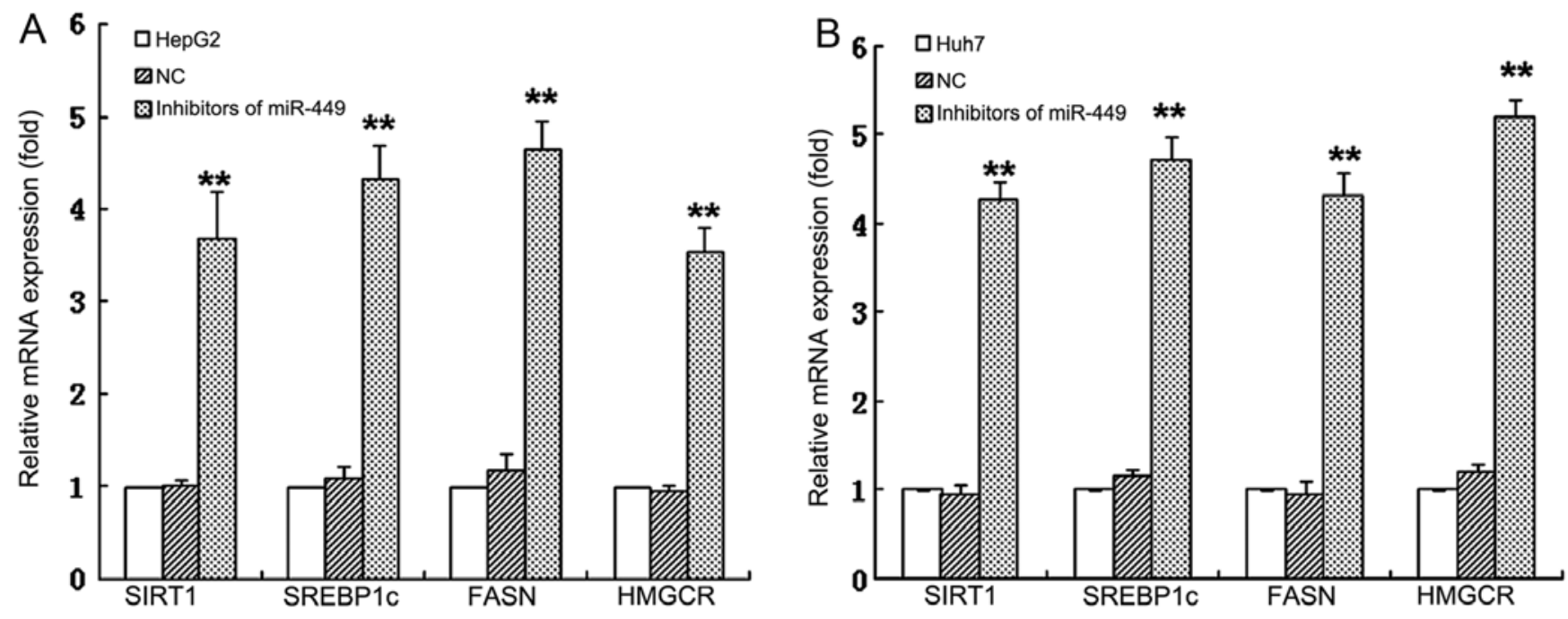

Figure 2. The inhibitors of miR-449 upregulated mRNA expression of SIRT1, SREBP1-c and their downstream genes in hepatoma cell lines. The mRNA expression of SREBP, FASN and HMGCR, which are downstream regulated genes of SIRT1, was increased in mRNA levels of (A) HepG2 and (B) Huh7 cells by inhibitors of miR-449. 

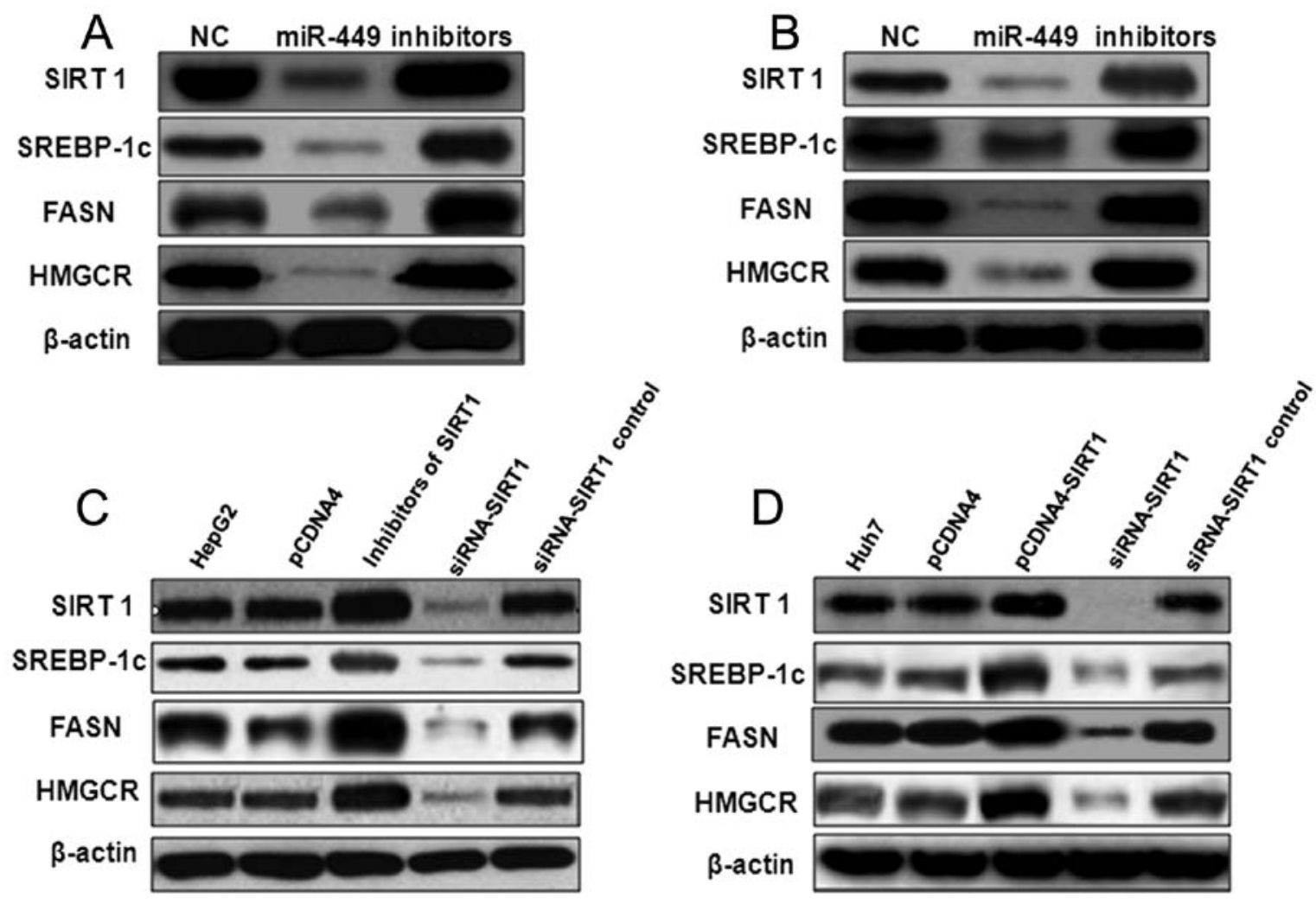

Figure 3. MiR-449 decreased the protein expression of SIRT1, SREBP1-c and their downstream genes in hepatoma cell lines. The protein level of SIRT1, SREBP-1c, FASN and HMGCR was inhibited by miR-449 in (A) HepG2 and (B) Huh7 also similarly to treatment with SIRT1 siRNA (C and D), but were upregulated by inhibitors of miR-449 or overexpression of SIRT1.
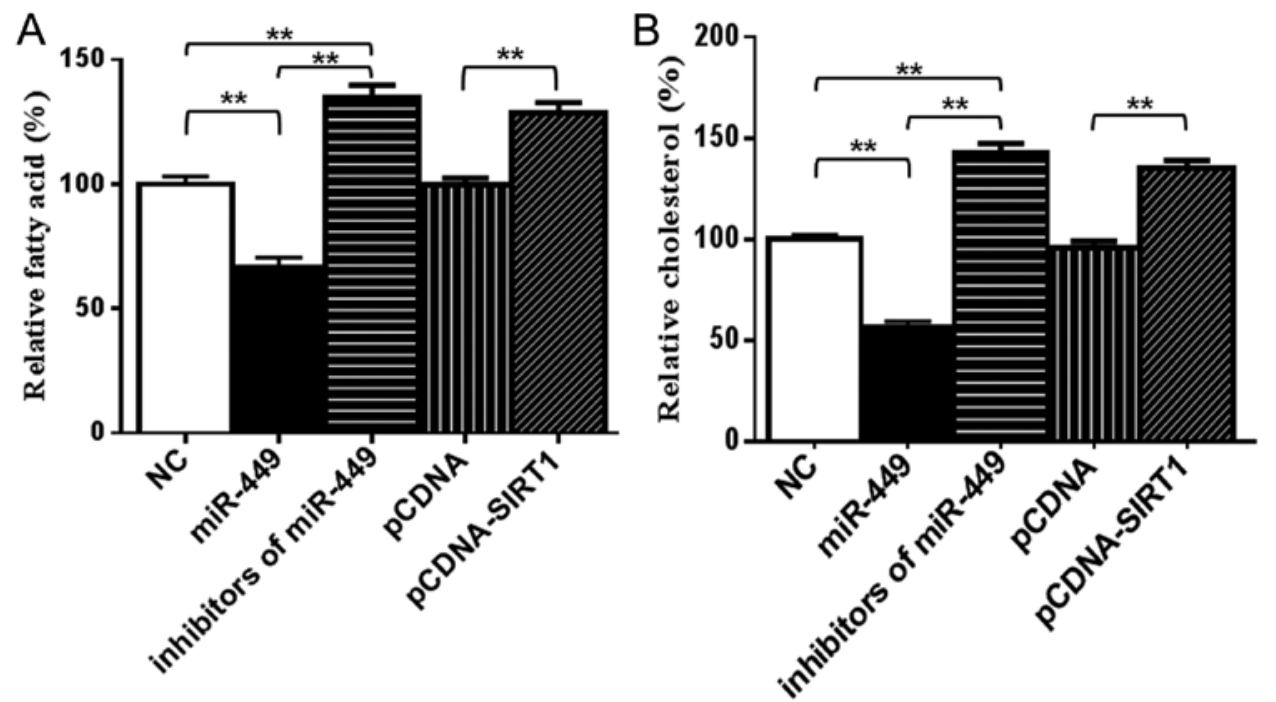

Figure 4. MiR-449 decreased the amounts of fatty acid and cholesterol in hepatoma cells. The amounts of intracellular fatty acid and cholesterol were significantly decreased in (A) miR-449-transfected HepG2 and (B) Huh7 cells compared with the control groups. To further test the specificity of miR-449 for SREBPlipogenesis-cholesterogenesis, antisense oligonucleotides against miR-449 were used as miR-449 inhibitors. By blocking endogenous miR-449 in hepatoma cells, the amounts of intracellular fatty acid and cholesterol were upregulated by inhibitors of miR-449 similarly to cells treated with overexpression of SIRT1 (A and B).

miR-449 by XTT assays, we used mimics of miR-449 in HepG2 and Huh7 cells and performed a series of functional assays relevant to tumorigenicity and cancer progression. When HepG2 (Fig. 7A) and Huh7 (Fig. 7B) cells were transfected with miR-449, proliferation of both cell types was inhibited in comparison with miR-NC cells. Conversely, by blocking miR-449 in hepatoma cells, miR-449 inhibitors increased cell proliferation (Fig. 7A and B). These data suggest that miR-449 suppresses the pathways relevant to tumorigenicity and cancer progression. 

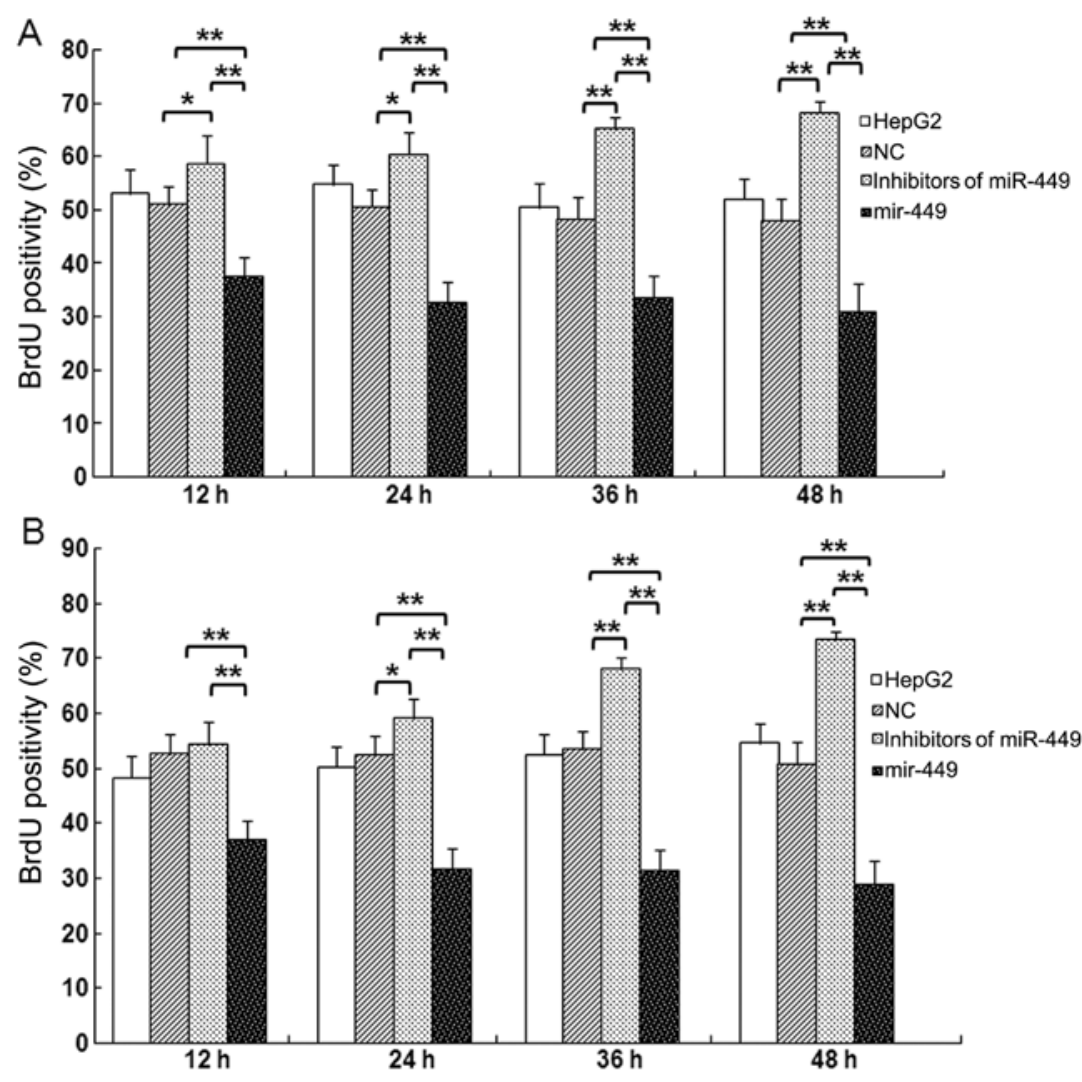

Figure 5. MiR-449 suppresses DNA synthesis in hepatoma cell lines. Cells were synchronized at the G1/S boundary by double thymidine block, and then released into mitosis. After $24 \mathrm{~h}, \mathrm{BrdU}$ was added into the medium at indicated time points to evaluate DNA synthesis. (A and B) Incorporation of BrdU into the control, accumulation of mitotic HepG2 and Huh7 cells was significantly delayed in miR-449-treated cells in each point. In contrast, accumulation of mitotic HepG2 or Huh7 cells was significantly promoted at every time point by inhibitors of miR-449.

\section{Discussion}

Sterol regulatory element-binding protein (SREBP) is a basic helix-loop-helix leucine zipper transcription factor with important metabolic roles in lipogenesis and cholesterogenesis (38-40). Three major SREBP isoforms have been identified, SREBP-1a, SREBP-1c and SREBP-2 (41). SREBP-1 controls genes involved in fatty acid, lipid and cholesterol biosynthesis (42), whereas SREBP-2 more specifically regulates cholesterol metabolism and homeostasis (45). Dysregulation of SREBPs and their downstream targeted genes associated with lipogenesis and cholesterogenesis has been implicated in cancer. Examples include fatty acid synthase (FASN), a metabolic oncogene $(44,45)$, and 3-hydroxy-3-methylglutaryl CoA reductase (HMGCR), the rate-limiting step in cholesterol biosynthesis; both proteins have been reported to be involved in the development and progression of cancer (44,46-49). Targeting the aberrant SREBP-lipogenesis-cholesterogenesis pathway may lead to new approaches to the treatment of cancer. Altered lipid metabolism has been recently linked to HCC pathogenesis.

Upregulation of lipogenesis and cholesterogenesis in cancer cells is associated with increased need for cell membrane components and activation of lipid raft-related intercellular signaling transduction during uncontrolled cell proliferation and division as well as cancer development and progression (50-53). Blockade of abnormal lipogenesis and cholesterogenesis provides a promising therapeutic approach for prevention or treatment of prostatic malignancy. MiRNA has been reported to regulate multiple important biological processes including metabolism $(54,55)$ and is of potential use in cancer therapy (56-58). However, how miRNA mediates aberrant fat metabolism and homeostasis in hepatoma cells remains unclear. In this study, we identified one miRNAs that play an important role in the regulation of lipogenesis and cholesterogenesis in hepatoma cells. MiR-449 inhibited fatty acid and cholesterol biosynthesis through downregulation of SIRT1 and key lipogenic or cholesterogenic transcription factors, SREBP-1c, and their downstream regulated genes including FASN and HMGCR.

SIRT1 also regulates metabolism and modulates metabolic diseases such as diabetes. Cellular studies showed that SIRT1 modulates fat accumulation, regulates mitochondrial biogenesis and activates fatty acid oxidation (59). Despite extensive studies for several decades, the role of SIRT1 in human cancer remains controversial. Increased SIRT1 expression has been found to reduce tumor formation in a mouse model of colon tumor $(60,61)$, whereas SIRT1 mutant mice have exhibited increased DNA instability and are more susceptible to tumor development (62). Reduced levels of SIRT1 mRNA and proteins are observed in breast tumors when compared with normal tissue (62). Intriguingly, however, SIRT1 overexpression is found in prostate (63), ovarian (64), gastric (65) and colorectal cancers (66), suggesting a role in tumorigenesis. Therefore, the function of SIRT1 may be tumor-type specific and also depend on 

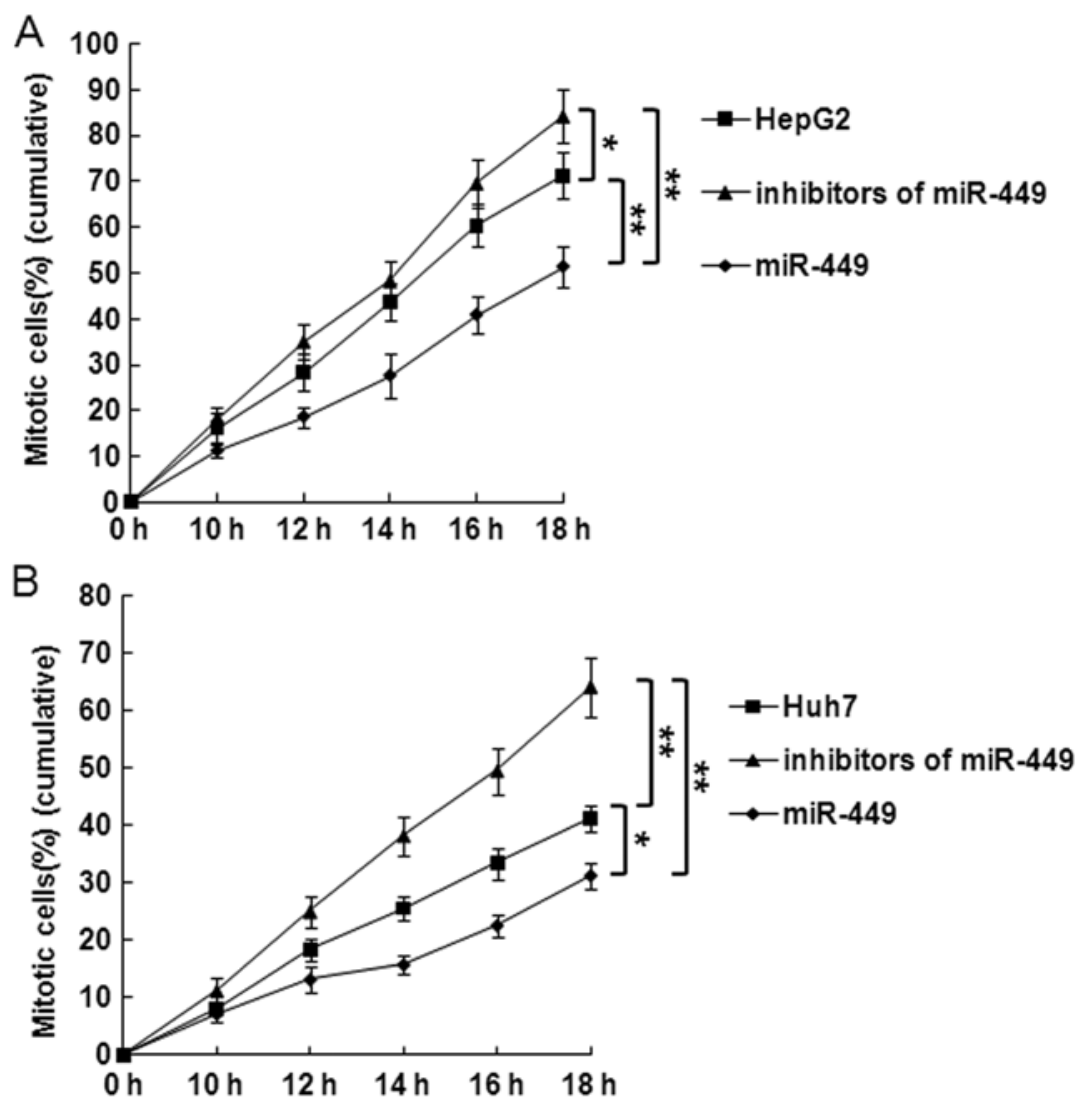

Figure 6. MiR-449 induces delay of mitotic entry in hepatoma cell lines. To further examine the specific effect of the over expression of miR-449 on mitotic entry, we repeated this experiment and evaluated the mitotic entry. Overexpression miR-449 significantly delayed mitotic entry of HepG2 or Huh7 cells (A and B). In contrast, mitotic entry of HepG2 or Huh7 cells was significantly promoted in miR-449-treated cells in each point (A and B).
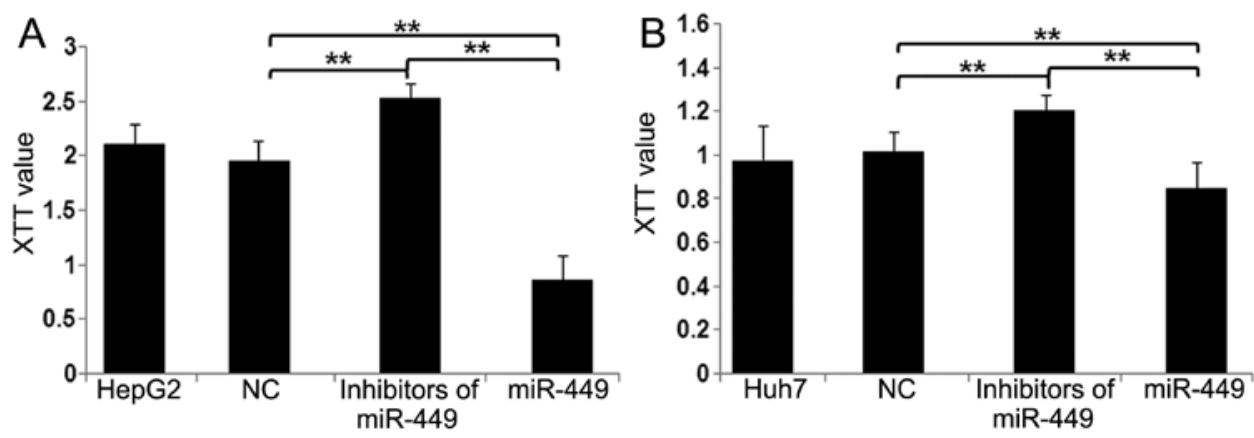

Figure 7. MiR-449 restrains cell proliferation of hepatoma cell lines. To assess the potential for biological consequences elicited by miR-449 by XTT assays, we used mimics of miR-449 in HepG2 and Huh7 cells and performed a series of functional assays relevant to tumorigenicity and cancer progression. When (A) HepG2 and (B) Huh7 cells were transfected with miR-449, proliferation of both cell types was inhibited in comparison with miR-NC cells. Conversely, by blocking miR-449 in hepatoma cells, miR-449 inhibitors increased cell proliferation (A and B).

the stage of tumorigenesis being assessed. In liver cancer, especially in HCC, Wang et al (62) used pooled microarray data from HCC samples to suggest that SIRT1 mRNA is expressed at comparable levels in both malignant and benign tissues. They further concluded that SIRT1 protein expression is reduced in HCC on the basis of one paired HCC specimen (61). In contrast, Chen et al (67) have demonstrated that SIRT1 protein was overexpressed by a post-transcriptional mechanism in a subset of HCC. They have also shown that SIRT1 expression is low in normal and premalignant livers, and its overexpression is closely associated with poorly differentiated histology (67). In agreement with the results of Bae et al, their study confirmed that SIRT1 protein was overexpressed in a subset of patients with HCC, whereas SIRT1 mRNA levels did not differ between $\mathrm{HCC}$ and non-tumoral tissues in a large cohort of HCC patients. Furthermore, they demonstrated that SIRT1 inactivation suppressed in vitro cell growth and proliferation of liver cancer cells. Many previous reports have suggested that histone deacetylase (HDAC)-mediated gene suppression can 
cause uncontrolled cell growth because HDACs repress the transcription of cyclin-dependent kinase inhibitors, allowing continued cellular proliferation $(68,69)$. These results clearly support the suggestion of oncogenic SIRT1 in hepatocellular malignant proliferation and transformation. However, regulation of SIRT1 activity or expression leading to oncogenic SIRT1 overexpression has not been reported yet.

Recent studies have suggested that SIRT1 expression is regulated by miRNAs in various tissues, and miRNAs regulating SIRT1 are involved in many cellular pathways, including embryonic stem cell differentiation, apoptosis, senescence and hypoxia. These findings led us to hypothesize that SIRT1 overexpression at the post-transcriptional level may be induced by the loss or suppression of a miRNA that selectively targets SIRT1. The induction of SIRT1 protein by Dicer knockdown helped us to identify endogenous miRNAs that blocked SIRT1 mRNA translation in HCC cells. In HCC cells, upregulation of HDAC1-3 reduces expression of miR-449. MiR-449 binds c-MET mRNA to reduce its levels, promoting apoptosis and reducing proliferation of liver cells. Expression of miR-449 slows growth of HCC xenograft tumors in mice; this miR might function as a tumor suppressor (70).

We have predicted SIRT1 as miR-449 target gene. SIRT1 could regulate SREBP-1c expression and activity in liver. SREBP-1 and FASN have been shown to be a potentially oncogenic transcription factor (71) and a metabolic oncogene (72,73), respectively. By integrative analyses, Li et al not only identified the miRNA predictors of survival, but also analysed the functional roles of miRNAs in the context of hepatoma progression. In conclusion, systematic analysis of the miRNA-mRNA regulatory network (FMRN) provides new insights into post-transcriptional gene regulation in the progression of hepatoma (74). The authors focused on the identification of the miRNA signatures associated with the hepatoma progression by considering their topological central roles in the FMRN. Similarly, the 'in-degree' for genes measured their central roles in the FMRN. By computing the correlation between in-degree of gene in the FMRN and the negative log-transformed P-value of survival analysis, in-degree is significantly positively associated with the power for survival analysis. These results suggest that the in-degree may be a good indicator for identifying the genes involved in the progression of hepatomas, extending the application of the integrative method.

We confirmed that SIRT1 mRNAs are direct targets of miR-449. The miR-449 inhibited the expression of SIRT1, SREBP-1c and their downstream gene FASN, HMGCR in HepG2 and Huh7 hepatoma cell lines, respectively. Our study suggested that miR-449 could inhibit SIRT1-SREBP signaling and DNA synthesis, mitotic entry, cell proliferation through reduction expression of SIRT1 and decreased the levels of fatty acid and cholesterol in hepatoma cells.

In summary, our study demonstrates for the first time that MiR-449 inhibits the expression of SIRT1 and SREBP-1c as well as their downstream regulated genes, and re-programs lipogenesis and cholesterogenesis in hepatoma cells. MiR-449 suppresses tumorigenicity and cell proliferation, which suggests that MiR-449 play a tumor-suppressive role in hepatoma. Collectively, miR-449 directly or indirectly regulates a cohort of genes with significant biological roles in lipid and cholesterol anabolism and homeostasis, cell proliferation and progression in hepatoma cells. The small non-coding RNA therefore provides potential therapeutic agents for treatment of hepatoma malignancy.

\section{Acknowledgements}

This study was supported by grants from the National Natural Science Foundation of China (nos. 30600524, 81341067, 81071990 and 81201758), Science and Technology Planning Project of Guangdong Province (nos. 2012A030400055, 2010B080701088, 2011B080701096 and 2011B031800184), Science and Technology projects of Guangzhou (nos. 2011J410010 and 2011J4300066), Special Project of Beijing Health Development and Scientific Research, China (no. 2011-5041-02), Key Projects in the PLA's logistics Program during the Twelfth Five-year Plan Period (no. BWS11J029). The study sponsors had no involvement in the study.

\section{References}

1. Herold C, Reck T, Fischler P, et al: Prognosis of a large cohort of patients with hepatocellular carcinoma in a single European centre. Liver 22: 23-28, 2002.

2. Okuda K: Hepatocellular carcinoma. J Hepatol 32: 225-237, 2000.

3. Bhalla KN: Epigenetic and chromatin modifiers as targeted therapy of hematologic malignancies. J Clin Oncol 23: 3971-3993, 2005.

4. Dowman JK, Hopkins LJ, Reynolds GM, al: Development of hepatocellular carcinoma in a murine model of nonalcoholic steatohepatitis induced by use of a high-fat/fructose diet and sedentary lifestyle. Am J Pathol 184: 1550-1561, 2014.

5. Karagozian R, Derdák Z and Baffy G: Obesity-associated mechanisms of hepatocarcinogenesis. Metabolism 63: 607-617, 2014.

6. Qin H and Ruan ZH: The role of monoacylglycerol lipase (MAGL) in the cancer progress. Cell Biochem Biophys: Mar 16, 2014 (Epub ahead of print).

7. Pralhada Rao R, Vaidyanathan N, Rengasamy M, Mammen Oommen A, Somaiya $\mathrm{N}$ and Jagannath MR: Sphingolipid metabolic pathway: an overview of major roles played in human diseases. J Lipids 2013: 178910, 2013.

8. Lee RC, Feinbaum RL and Ambros V: The C. elegans heterochronic gene lin-4 encodes small RNAs with antisense complementarity to lin-14. Cell 75: 843-854, 1993.

9. Xu P, Vernooy SY, Guo M and Hay BA: The Drosophila microRNA Mir-14 suppresses cell death and is required for normal fat metabolism. Curr Biol 13: 790-795, 2003.

10. Wightman B, Ha I and Ruvkun G: Post-transcriptional regulation of the heterochronic gene lin-14 by lin- 4 mediates temporal pattern formation in C.elegans. Cell 75: 855-862, 1993.

11. Reinhart BJ, Slack FJ, Basson M, et al: The 21-nucleotide let-7 RNA regulates developmental timing in Caenorhabditis elegans. Nature 403: 901-906, 2000

12. Brennecke J, Hipfner DR, Stark A, Russell RB and Cohen SM: Bantam encodes a developmentally regulated microRNA that controls cell proliferation and regulates the proapoptotic gene hid in Drosophila. Cell 113: 25-36, 2003.

13. Hatfield SD, Shcherbata HR, Fischer KA, Nakahara K, Carthew RW and Ruohola-Baker H: Stem cell division is regulated by the microRNA pathway. Nature 435: 974-978, 2005.

14. Michael MZ, O' Connor SM, van Holst Pellekaan NG, Young GP and James RJ: Reduced accumulation of specific microRNAs in colorectal neoplasia. Mol Cancer Res 1: 882-891, 2003.

15. Calin GA, Sevignani C, Dumitru CD, et al: Human microRNA genes are frequently located at fragile sites and genomic regions involved in cancers. Proc Natl Acad Sci USA 101: 2999-3004, 2004.

16. Iorio MV, Ferracin M, Liu CG, et al: MicroRNA gene expression deregulation inhumanbreast cancer. Cancer Res 65: 7065-7070, 2005. 
17. Lu J, Getz G, Miska EA, et al: MicroRNA expression profiles classify human cancers. Nature 435: 834-838, 2005.

18. He L, Thomson JM, Hemann MT, et al: A microRNA polycistron as a potential human oncogene. Nature 435: 828-833, 2005

19. Volinia S, Calin GA, Liu CG, et al: A microRNA expression signature of human solid tumors defines cancer gene targets. Proc Natl Acad Sci USA 103: 2257-2261, 2006.

20. Pekarsky Y, Santanam U, Cimmino A, et al: Tcl1 expression in chronic lymphocytic leukemia is regulated by miR-29 and miR-181. Cancer Res 66: 11590-11593, 2006.

21. Slack FJ and Weidhaas JB: MicroRNAs as a potential magic bullet in cancer. Future Oncol 2: 73-82, 2006.

22. Cimmino A, Calin GA, Fabbri M, et al: miR-15 and miR-16 induce apoptosis by targeting BCL2. Proc Natl Acad Sci USA 102: 13944-13949, 2005.

23. Johnson SM, Grosshans $\mathrm{H}$, Shingara $\mathrm{J}$, et al: RAS is regulated by the let-7 microRNA family. Cell 120: 635-647, 2005.

24. Takamizawa J, Konishi H, Yanagisawa K, et al: Reduced expression of the let-7 microRNAs in human lung cancers in association with shortened postoperative survival. Cancer Res 64: 3753-3756, 2004.

25. Mayr C, Hemann MT and Bartel DP: Disrupting the pairing between let-7 and Hmga2 enhances oncogenic transformation. Science 315: 1576-1579, 2007.

26. Morris JP and McManus MT: Slowing down the Ras lane: miRNAs as tumor suppressors? Sci STKE 16: 41, 2005

27. He L, He X, Lowe SW and Hannon GJ: MicroRNAs join the p53 network - another piece in the tumour-suppression puzzle Nat Rev Cancer 7: 819-822, 2007.

28. Lizé M, Klimke A and Dobbelstein M: MicroRNA-449 in cell fate determination. Cell Cycle 10: 2874-2882, 2011.

29. Lizé M, Pilarski S and Dobbelstein M: E2F1-inducible microRNA 449a/b suppresses cell proliferation and promotes apoptosis. Cell Death Differ 17: 452-458, 2010.

30. Hida Y, Kubo Y, Murao K and Arase S: Strong expression of a longevity-related protein, SIRT1, in Bowen's disease. Arch Dermatol Res 299: 103-106, 2007.

31. Yeung F, Hoberg JE, Ramsey CS, et al: Modulation of NF-kappaB-dependent transcription and cell survival by the SIRT1 deacetylase. EMBO J 23: 2369-2380, 2004

32. Walker AK, Yang F, Jiang K, et al: Conserved role of SIRT1 orthologs in fasting-dependent inhibition of the lipid/cholesterol regulator SREBP. Genes Dev 24: 1403-1417, 2010.

33. Rodgers JT and Puigserver P: Fasting-dependent glucose and lipid metabolic response through hepatic sirtuin 1. Proc Natl Acad Sci USA 104: 12861-12866, 2007.

34. Ponugoti $\mathrm{B}, \mathrm{Kim} \mathrm{DH}, \mathrm{Xiao} \mathrm{Z}$, et al: SIRT1 deacetylates and inhibits SREBP-1C activity in regulation of hepatic lipid metabolism. J Biol Chem 285: 33959-33970, 2010.

35. Truman JP, García-Barros M, Obeid LM and Hannun YA: Evolving concepts in cancer therapy through targeting sphingolipid metabolism. Biochim Biophys Acta: Dec 30, 2013 (Epub ahead of print).

36. Zhou B, Li C, Qi W, et al: Downregulation of miR-181a upregulates sirtuin-1 (SIRT1) and improves hepatic insulin sensitivity. Diabetologia 55: 2032-2043, 2012.

37. Ford J, Jiang M and Milner J: Cancer-specific functions of SIRT1 enable human epithelial cancer cell growth and survival. Cancer Res 65: 10457-10463, 2005.

38. Hamamoto R, Furukawa Y, Morita M, et al: SMYD3 encodes a histone methyltransferase involved in the proliferation of cancer cells. Nat Cell Biol 6: 731-740, 2004.

39. Wang $\mathrm{H}$, Liu $\mathrm{H}$, Chen $\mathrm{K}$, et al: SIRT1 promotes tumorigenesis of hepatocellular carcinoma through PI3K/PTEN/AKT signaling. Oncol Rep 28: 311-318, 2012.

40. Eberle D, Hegarty B, Bossard P, Ferre P and Foufelle F: SREBP transcription factors: master regulators of lipid homeostasis Biochimie 86: 839-848, 2004

41. Dif N, Euthine V, Gonnet E, et al: Insulin activates human sterol-regulatory-element-binding protein-1c (SREBP-1c) promoter through SRE motifs. Biochem J 400: 179-188, 2006.

42. Guillet-Deniau I, Mieulet V, Le Lay S, et al: Sterol regulatory element binding protein-1c expression and action in rat muscles: insulin-like effects on the control of glycolytic and lipogenic enzymes and UCP3 gene expression. Diabetes 51: 1722-1728, 2002.

43. Rome S, Lecomte V, Meugnier E, et al: Microarray analyses of SREBP-1a and SREBP-1c target genes identify new regulatory pathways in muscle. Physiol Genomics 34: 327-337, 2008.
44. Giandomenico V, Simonsson M, Gronroos E and Ericsson J: Coactivator dependent acetylation stabilizes members of the SREBP family of transcription factors. Mol Cell Biol 23: 2587-2599, 2003

45. Calkin AC and Tontonoz P: Transcriptional integration of metabolism by the nuclear sterol-activated receptors LXR and FXR. Nat Rev Mol Cell Biol 13: 213-224, 2012.

46. Yoshikawa $\mathrm{T}$, Shimano $\mathrm{H}$, Amemiya-Kudo $\mathrm{M}$, et al: Identification of liver $X$ receptor-retinoid $X$ receptor as an activator of the sterol regulatory element-binding protein $1 \mathrm{c}$ gene promoter. Mol Cell Biol 21: 2991-3000, 2001.

47. Repa JJ, Liang G, Ou J, et al: Regulation of mouse sterol regulatory element-binding protein-1c gene (SREBP-1c) by oxysterol receptors, LXRalpha and LXRbeta. Genes Dev 14: 2819-2830, 2000.

48. Cozzone D, Debard C, Dif N, et al: Activation of liver X receptors promotes lipid accumulation but does not alter insulin action in human skeletal muscle cells. Diabetologia 49: 990-999, 2006

49. Swinnen JV, Heemers $H$, van de Sande T, et al: Androgens, lipogenesis and prostate cancer. J Steroid Biochem Mol Biol 92: 273-279, 2004

50. Yamashita T, Honda M, Takatori $\mathrm{H}$, et al: Activation of lipogenic pathway correlates with cell proliferation and poor prognosis in hepatocellular carcinoma. J Hepatol 50: 100-110, 2009.

51. Di Vizio D, Solomon KR and Freeman MR: Cholesterol and cholesterol-rich membranes in prostate cancer: an update. Tumori 94: 633-639, 2008.

52. Swinnen JV, Brusselmans K and Verhoeven G: Increased lipogenesis in cancer cells: new players, novel targets. Curr Opin Clin Nutr Metab Care 9: 358-365, 2006.

53. Freeman MR, Cinar B and Lu ML: Membrane rafts as potential sites of nongenomic hormonal signaling in prostate cancer. Trends Endocrinol Metab 16: 273-279, 2005.

54. Fernandez-Hernando C, Suarez Y, Rayner KJ and Moore KJ: MicroRNAs in lipid metabolism. Curr Opin Lipidol 22: 86-92, 2011.

55. Krutzfeldt J and Stoffel M: MicroRNAs: a new class of regulatory genes affecting metabolism. Cell Metab 4: 9-12, 2006.

56. Henry JC, Azevedo-Pouly AC and Schmittgen TD: MicroRNA replacement therapy for cancer. Pharm Res 28: 3030-3042, 2011.

57. Garzon R, Marcucci G and Croce CM: Targeting microRNAs in cancer: rationale, strategies and challenges. Nat Rev Drug Discov 9: 775-789, 2010.

58. Bader AG, Brown D and Winkler M: The promise of microRNA replacement therapy. Cancer Res 70: 7027-7030, 2010

59. Lee J and Kemper JK: Controlling SIRT1 expression by microRNAs in health and metabolic disease. Aging (Albany, NY) 2: 527-534, 2010 .

60. Firestein R, Blander G, Michan S, et al: The SIRT1 deacetylase suppresses intestinal tumorigenesis and colon cancer growth. PLoS One 3: e2020, 2008.

61. Bae HJ, Noh JH, Kim JK, et al: MicroRNA-29c functions as a tumor suppressor by direct targeting oncogenic SIRT1 in hepatocellular carcinoma. Oncogene 33: 2557-2567, 2014.

62. Wang RH, Sengupta K, Li C, et al: Impaired DNA damage response, genome instability, and tumorigenesis in SIRT1 mutant mice. Cancer Cell 14: 312-323, 2008

63. Huffman DM, Grizzle WE, Bamman MM, et al: SIRT1 is significantly elevated in mouse and human prostate cancer. Cancer Res 67: 6612-6618, 2007.

64. Jang KY, Kim KS, Hwang SH, et al: Expression and prognostic significance of SIRT1 in ovarian epithelial tumours. Pathology 41: 366-371, 2009.

65. Cha EJ, Noh SJ, Kwon KS, et al: Expression of DBC1 and SIRT1 is associated with poor prognosis of gastric carcinoma. Clin Cancer Res 15: 4453-4459, 2009.

66. Stunkel W, Peh BK, Tan YC, et al: Function of the SIRT1 protein deacetylase in cancer. Biotechnol J 2: 1360-1368, 2007.

67. Chen J, Zhang B, Wong N, et al: Sirtuin 1 is upregulated in a subset of hepatocellular carcinomas where it is essential for telomere maintenance and tumor cell growth. Cancer Res 71: 4138-4149, 2011.

68. Noh JH, Jung KH, Kim JK, et al: Aberrant regulation of HDAC2 mediates proliferation of hepatocellular carcinoma cells by deregulating expression of G1/S cell cycle proteins. PLoS One 6: e28103, 2011. 
69. Xie HJ, Noh JH, Kim JK, et al: HDAC1 inactivation induces mitotic defect and caspase-independent autophagic cell death in liver cancer. PLoS One 7: e34265, 2012.

70. Buurman R, Gürlevik E, Schäffer V, et al: Histone deacetylases activate hepatocyte growth factor signaling by repressing microRNA-449 in hepatocellular carcinoma cells. Gastroenterology 143: 811-820, 2012.

71. Huang WC, Li X, Liu J, Lin JT and Chung LW: Activation of androgen receptor, lipogenesis and oxidative stress converged by SREBP-1 is responsible for regulating growth and progression of prostate cancer cells. Mol Cancer Res 10: 133-142, 2012.
72. Menendez JA, Decker JP and Lupu R: In support of fatty acid synthase (FAS) as a metabolic oncogene: extracellular acidosis acts in an epigenetic fashion activating FAS gene expression in cancer cells. J Cell Biochem 94: 1-4, 2005.

73. Baron A, Migita T, Tang D and Loda M: Fatty acid synthase: a metabolic oncogene in prostate cancer? J Cell Biochem 91: 47-53, 2004.

74. Li Y, Xu J, Chen H, et al: Comprehensive analysis of the functional microRNA-mRNA regulatory network identifies miRNA signatures associated with hepatoma malignant progression. Nucleic Acids Res 41: e203, 2013. 